\title{
Hypofractionated Hybrid Radiotherapy Techniques for Synchronous Bilateral Breast Cancer
}

\author{
Karunakaran Balaji ${ }^{1,2}$, Velayudham Ramasubramanian ${ }^{1 *}$
}

\begin{abstract}
Objective: This study intends at a dosimetric comparison of four different hybrid plans which is a combination of different radiation therapy techniques, for synchronous bilateral breast cancer (SBBC) irradiation using a hypofractionated dose regimen. Methods: Four different hybrid techniques: 3DCRT+IMRT, 3DCRT+VMAT, IMRT+VMAT, and VMAT+IMRT were planned using computed tomography (CT) images of fifteen SBBC patients. All hybrid plans were generated using a hypofractionated dose prescription of $40.5 \mathrm{~Gy}$ in 15 fractions. $70 \%$ of the dose was planned with a base-dose component and the remaining $30 \%$ of the dose was planned with a hybrid component. The plans were evaluated based on the PTV and organs at risk (OARs) dosimetric parameter results and computed a plan quality score for each plan. Results: The results for PTV parameters have shown that the 3DCRT+VMAT and 3DCRT+IMRT plans were better than other plans. The $3 \mathrm{DCRT} \pm \mathrm{IMRT}$ plan was provided better results for OARs, while IMRT $\pm \mathrm{VMAT}$ and VMAT+IMRT plans were increased the low dose volumes to the heart and lungs. The 3DCRT+VMAT plan was required less monitor units and treatment time compared to other plans. Conclusion: The overall plan quality score that integrated the dosimetric parameters of PTV and OARs indicated that the 3DCRT+VMAT hybrid plan is superior for SBBC treatment.
\end{abstract}

Keywords: Bilateral breast cancer- hybrid plans- IMRT- VMAT- hypofractionation

Asian Pac J Cancer Prev, 22 (12), 3933-3939

\section{Introduction}

Synchronous bilateral breast cancer $(\mathrm{SBBC})$ is a rare entity defined as tumors occurring concurrently in both breasts. Though the incidence of SBBC is lower compared to that of unilateral breast cancer, the burden of disease is relatively high in terms of complications and survival (Kheirelseid et al., 2011). As like unilateral breast cancer, adjuvant radiation therapy (RT) is an integral part of the multi modal management of SBBC patients. However, RT of SBBC is more complex, owing to multiple fields for a wider treatment volume and with the irradiation of both the lungs and heart. Additionally, the reduction of RT-related toxicities to the lungs and heart as well as the better cosmetic outcome of breasts are highly anticipated.

The accelerated hypofractionated RT for the whole breast (WB) or chest wall $(\mathrm{CW})$ with reduced course duration (approximately 3 weeks) is of recent clinical interest (Haviland et al., 2013; Badiyan et al., 2014; Khan et al., 2017). Furthermore, the hypofractionated RT widens the therapeutic ratio. In a recent clinical study, Gadea et al., (2021) evaluated the feasibility, safety, and toxicity of hypofractionated RT (40.05 Gy in 15 fractions) for SBBC and showed better clinical outcomes in comparison with conventional fractionation (50 Gy in 25 fractions).
Similarly, other clinical studies showed the feasibility and satisfactory early toxicity in the hypofractionated RT for SBBC (Scorsetti et al., 2012; Narasimhulu et al., 2020). Dosimetric comparison studies that utilizing hypofractionated dose regimens for SBBC are sparse.

Three-dimensional conformal radiotherapy (3DCRT) is a standard treatment technique for breast cancer RT which provides a reduced dose to organs at risk (OARs). However, the inclusion of both breasts extensively irradiates the heart and both lungs using the standard 3DCRT technique. Further, the 3DCRT technique required greater care at the midline area between both breasts to avoid high dose spread due to overlying fields. Intensity modulated radiation therapy (IMRT) and volumetric modulated arc therapy (VMAT) provide conformal and homogeneous doses to the SBBC planning target volume (PTV). These advanced techniques reduce the high dose irradiation to normal tissues (NT) while delivering increased low dose spread to the lungs and heart. In this context, contribution of the modulated beams is highly appreciable along with the 3DCRT fields to attain balanced results (Balaji et al., 2016)

A novel hybrid technique is a blend of 3DCRT and IMRT/VMAT with different dose proportions that simultaneously deliver in each treatment fraction. 
Improvement in the dosimetric parameter results of hybrid plans for unilateral breast cancer has been described in many articles (Jost et al., 2015; Lin et al., 2015; Chen et al., 2017). While there is a paucity of hybrid planning studies that include SBBC. Most of the hybrid studies employed the 3DCRT as a base-dose plan for the VMAT or IMRT to generate the hybrid plans, while few studies utilized the IMRT as a base-dose plan with flattening filter free (FFF) beams (Bahrainy et al., 2016). Publications that compare hybrid plans with different combinations of techniques together are scarce.

The aim of this study is a dosimetric assessment of four different hybrid planning techniques: 3DCRT (base-dose plan) combined with IMRT (3DCRT+IMRT), 3DCRT (base-dose plan) combined with VMAT (3DCRT+VMAT), IMRT (base-dose plan) combined with VMAT (IMRT+VMAT) and VMAT (base-dose plan) combined with IMRT (VMAT+IMRT) for the SBBC RT using hypofractionated dose regimen.

\section{Materials and Methods}

\section{Patient preparation}

Computed tomography (CT) image set of fifteen SBBC patients who already received RT at our institution were retrospectively selected for this study. The median age of these patients was 52 years (range $49-66$ ). The informed consent has been waived off by the ethics board of the institution considering this as a retrospective study with no human involved. The planning CT images had been acquired with patients placed in a supine position using a customized vacuum bag. Their hands were elevated above the head and kept on a wing board. The CT data sets were imported in Eclipse treatment planning systems (TPS) version 13.7 (Varian Medical Systems, Palo Alto, CA, USA). Clinical target volume (CTV) that includes both breasts was delineated and expanded to $5 \mathrm{~mm}$ to generate PTV. The PTV was cropped $5 \mathrm{~mm}$ inside from the external body surface. The OARs delineated were heart, left anterior descending coronary artery (LAD), right coronary artery (RCA), left lung (LL), right lung (RL), spinal cord (SC), esophagus, trachea, gastroesophageal junction \& coeliac plexus (GEJCP), and NT, defined as the body volume minus PTV were delineated.

\section{Treatment Planning}

The hybrid treatment plans were created in the Eclipse TPS using $6 \mathrm{MV}$ photon beams of Truebeam STx linear accelerator (Varian Medical Systems, Palo Alto, CA, USA) furnished with a high-definition multi-leaf collimator (HDMLC). A hypofractionated dose prescription of 40.5 Gy (2.7 Gy per fraction) in 15 fractions was used for the PTV. The dose weightings utilized were $70 \%$ of the prescription dose for the base-dose component and 30\% of the prescription dose for the hybrid component (Balaji et al., 2018). Both IMRT and VMAT optimizations were performed using photon optimizer (PO) algorithm version 13.7. The volume dose was computed using Analytical Anisotropic Algorithm (AAA) version 13.7. with $2.5 \mathrm{~mm}$ dose grid matrix. All individual plans were normalized in such a way that the plans deliver the PTV mean dose equal to the prescribed dose. For all planning techniques, a single isocenter was placed longitudinally at the center of PTV and medially below the sternum as illustrated in Figure 1.

For the base-dose component, the 3DCRT plan employed two tangential fields for each side of the breast PTV as shown in Figure 1. The gantry angles of these tangential fields were selected in such a way that to avoid midline overlap between both sides of the PTV. A collimator angle of $0^{\circ}$ was kept for the tangential fields. Similarly, the IMRT base-dose plan was created using the same beam arrangements of the 3DCRT plan except for the collimator angles, which were kept around $\pm 10^{\circ}$ depends on the midline overlap between both sides of the PTV. In the optimization interface, fixed jaw setting was enabled for all IMRT fields. The VMAT base-dose plan included four tangential arcs for each breast as shown in Figure 1. For left-sided breast, arc1 rotated clockwise from $300^{\circ}$ to $350^{\circ}$ with $15^{\circ}$ collimator angle, arc 2 rotated clockwise from $100^{\circ}$ to $150^{\circ}$ with $345^{\circ}$ collimator angle, arc3 rotated counterclockwise from $150^{\circ}$ to $100^{\circ}$ with $350^{\circ}$ collimator angle, and arc 4 rotated counterclockwise from $350^{\circ}$ to $300^{\circ}$ with $10^{\circ}$ collimator angle. For right-sided breast, arc1 rotated clockwise from $210^{\circ}$ to $260^{\circ}$ with $15^{\circ}$ collimator angle, arc 2 rotated clockwise from $10^{\circ}$ to $60^{\circ}$ with $345^{\circ}$ collimator angle, arc3 rotated counterclockwise from $60^{\circ}$ to $10^{\circ}$ with $350^{\circ}$ collimator angle, and arc4 rotated counterclockwise from $260^{\circ}$ to $210^{\circ}$ with $10^{\circ}$ collimator angle. Both the IMRT and VMAT plan optimizations were performed using the photon optimizer (PO) algorithm. All base-dose plans were dose computed for $1.9 \mathrm{~Gy}$ (approximately $70 \%$ of prescription dose) using AAA.

For the hybrid component, IMRT and VMAT plans were generated with the remaining prescription dose of 0.8 Gy (approximately $30 \%$ of the PTVs prescription dose). The IMRT plans consisted of 5 fields for each breast as shown in Figure 2. The gantry angles for the left side are $300^{\circ}, 330^{\circ}, 45^{\circ}, 120^{\circ}$, and $150^{\circ}$. Similarly, the gantry angles for the right side are $60^{\circ}, 30^{\circ}, 315^{\circ}, 240^{\circ}$, and $210^{\circ}$. The collimator angles were set at $\pm 5^{\circ}$. The fixed jaw setting was enabled for all IMRT fields during optimization. The VMAT plans utilized two coplanar partial arcs and two tangential arcs for each breast as displayed in Figure 2. For the left side, arc1 rotated clockwise from $300^{\circ}$ to $45^{\circ}$ with $10^{\circ}$ collimator angle and arc 2 rotated clockwise from $45^{\circ}$ to $150^{\circ}$ with $350^{\circ}$ collimator angle. The tangential arc3 rotated counterclockwise from $150^{\circ}$ to $100^{\circ}$ with $345^{\circ}$ collimator angle and arc 4 rotated counterclockwise from $350^{\circ}$ to $300^{\circ}$ with $15^{\circ}$ collimator angle. For the right side, arc 1 rotated clockwise from $210^{\circ}$ to $315^{\circ}$ with $10^{\circ}$ collimator angle and arc 2 rotated clockwise from $315^{\circ}$ to $60^{\circ}$ with $350^{\circ}$ collimator angle. The tangential $\operatorname{arc} 3$ rotated counterclockwise from $60^{\circ}$ to $10^{\circ}$ with $345^{\circ}$ collimator angle and arc4 rotated counterclockwise from $260^{\circ}$ to $210^{\circ}$ with $15^{\circ}$ collimator angle. While doing optimization, the corresponding base-dose 3DCRT, IMRT, and VMAT plans were enabled. The optimization dose constraints were almost similar for all plans. After the volume dose calculation and normalization, plan sum of the 3DCRT+IMRT, 3DCRT+VMAT, IMRT+VMAT, and VMAT+IMRT hybrid plans were created. 


\section{Dosimetric evaluation}

The PTV and OARs dosimetric evaluation of these hybrid plans had been made using dose-volume histogram (DVH) analysis. For the PTV quality comparison, dosimetric indices like coverage index (COI), conformity index (CI), uniformity index (UI), and gradient index (GI) were calculated as stated below. The COI was defined as:

$$
\mathrm{COI}=\frac{\mathrm{D}_{P}}{\mathrm{D}_{95 \%}}
$$

where $\mathrm{D}_{\mathrm{P}}$ is the prescription dose and $\mathrm{D}_{95 \%}$ is the dose received by $95 \%$ of the PTV. The CI was calculated as:

$$
\mathrm{CI}=\frac{\mathrm{V}_{\text {PTV }}}{\mathrm{V}_{\text {PTVref }}} \times \frac{\mathrm{V}_{\text {ref }}}{\mathrm{V}_{\text {PTVref }}}
$$

where $\mathrm{V}_{\text {PTV }}$ is the volume of PTV, $\mathrm{V}_{\text {PTVref }}$ is the reference isodose (95\%) volume within the PTV, and $\mathrm{V}_{\text {ref }}$ is the volume of reference isodose $(95 \%)$. The UI was calculated as:

$$
\mathrm{UI}=\frac{\mathrm{D}_{5 \%}}{\mathrm{D}_{95 \%}},
$$

where $\mathrm{D}_{5 \%}, \mathrm{D}_{95 \%}$ are the doses received by $5 \%, 95 \%$ of the PTV respectively. The GI was defined as:

$$
\mathrm{GI}=\frac{\mathrm{V}_{50 \%}}{\mathrm{~V}_{P T V}}
$$

where $\mathrm{V}_{50 \%}$ is $50 \%$ isodose volume and $\mathrm{V}_{\text {PTV }}$ is the volume of PTV. The ideal value for COI, CI, UI, and GI is 1 and, a plan with a value closer to 1 indicates a superior plan. The dose-volume parameters evaluated for the comparison of OARs were listed in Table 1. In addition, total monitor units (MU) and treatment time (TT) were noted to assess the delivery efficiency.

A simple plan quality matrix scoring method was utilized to calculate an overall score that incorporates all dosimetric parameters evaluated. The overall score was calculated as:

\section{Overall Score $=\sum_{i=1}^{n}\left(\frac{\mathrm{A}_{i}}{\mathrm{D}_{i}}\right) X \mathrm{P}_{i}$}

where $\mathrm{Ai}$ is an achieved value of the ith dosimetric index of a particular plan and Di is the desired value ith dosimetric index and $\mathrm{n}$ is a number of dosimetric indices assessed. The Pi is a penalty function that doubles the score if the achieved value shows an unacceptable result (that is, more than desired value). For the COI, CI, UI, GI, MU, and TT their average values from all plans were taken as the desired values. Practically, the ideal value 1 cannot be achievable. Therefore the values between the ideal value and the desired value indicated the better plan. The desired values for the OARs are presented in Table 1. The overall score close to 0 designates a superior plan.

\section{Statistical analysis}

The dosimetric results of four different hybrid plans were analyzed using the Kruskal-Wallis test for multiple samples comparison. The statistical test was two-tailed, with a threshold for statistical significance of $p<0.05$.

\section{Results}

The mean \pm standard deviation (SD) of volumes of bilateral breast PTV, LL, RL, and heart were 1804.3 \pm 875.1 , $889.9 \pm 143.8,1147.8 \pm 204.9$, and $467.4 \pm 70.2 \mathrm{~cm}^{3}$ respectively. The PTV parameter results for all hybrid plans are summarized in Table 2. The hybrid plans achieved expected coverage $(\mathrm{COI}<1.05)$ for the PTV, while there were statistically significant differences among the plans $(\mathrm{p}=0.026)$. The UI of the PTV has shown statistical significance among all hybrid plans $(p=0.012)$. The CI and GI of the PTV have shown comparable results among all hybrid plans. The MU and

Table 1. Tolerance Dose Limits for All Organs at Risk

\begin{tabular}{ll}
\hline Organs at risk & Dose limits \\
\hline RL and LL & $\mathrm{V}_{5 \mathrm{~Gy}}<60 \% ; \mathrm{V}_{20 \mathrm{~Gy}}<30 \%$ \\
& $\mathrm{~V}_{35 \mathrm{~Gy}}<5 \% ; \mathrm{D}_{\text {Mean }}<12 \mathrm{~Gy}$ \\
Heart & $\mathrm{V}_{5 \mathrm{~Gy}}<40 \% ; \mathrm{V}_{25 \mathrm{~Gy}}<10 \%$ \\
& $\mathrm{~V}_{35 \mathrm{~Gy}}<5 \% ; \mathrm{D}_{\text {Mean }}<5 \mathrm{~Gy}$ \\
LAD & $\mathrm{V}_{30 \mathrm{~Gy}}<15 \% ; \mathrm{D}_{\text {Mean }}<15 \mathrm{~Gy}$ \\
RCA & $\mathrm{D}_{\text {Mean }}<4 \mathrm{~Gy}$ \\
Esophagus, Trachea & $\mathrm{D}_{\text {Mean }}<10 \mathrm{~Gy}$ \\
GEJCP & $\mathrm{D}_{\text {Mean }}<3 \mathrm{~Gy}$ \\
SC & $\mathrm{D}_{\text {Max }}<10 \mathrm{~Gy}$ \\
NT & $\mathrm{V}_{5 \mathrm{~Gy}}<20 \% ; \mathrm{D}_{\text {Mean }}<5 \mathrm{~Gy}$ \\
\hline
\end{tabular}

RL, Right lung; LL, Left lung; LAD, Left anterior descending coronary artery; RCA,Right coronary artery; GEJCP, Gastroesophageal junction \& coeliac plexus ; SC, Spinal cord; NT, Normal tissue; Gy, Gray; $\mathrm{V}_{\mathrm{XGY}}$, Volume receiving X Gy dose; $\mathrm{D}_{\text {Mean, }}$, Mean dose; $\mathrm{D}_{\text {Max }}$, Maximum dose
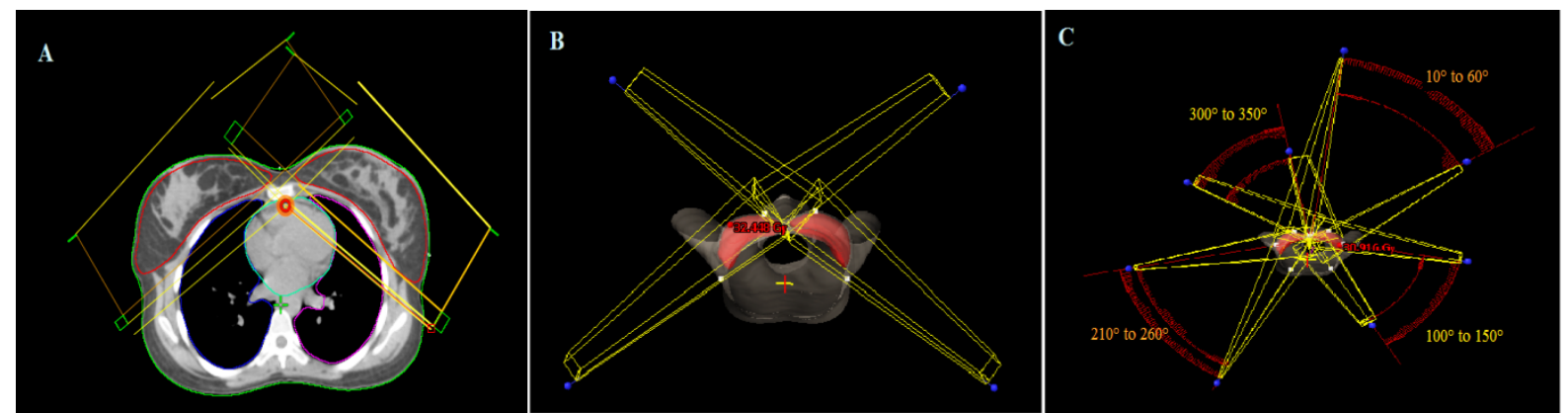

Figure 1. (a) Location of isocenter, (b) field arrangement in base-dose 3DCRT and IMRT plans, and (c) field arrangement in base-dose VMAT plan 

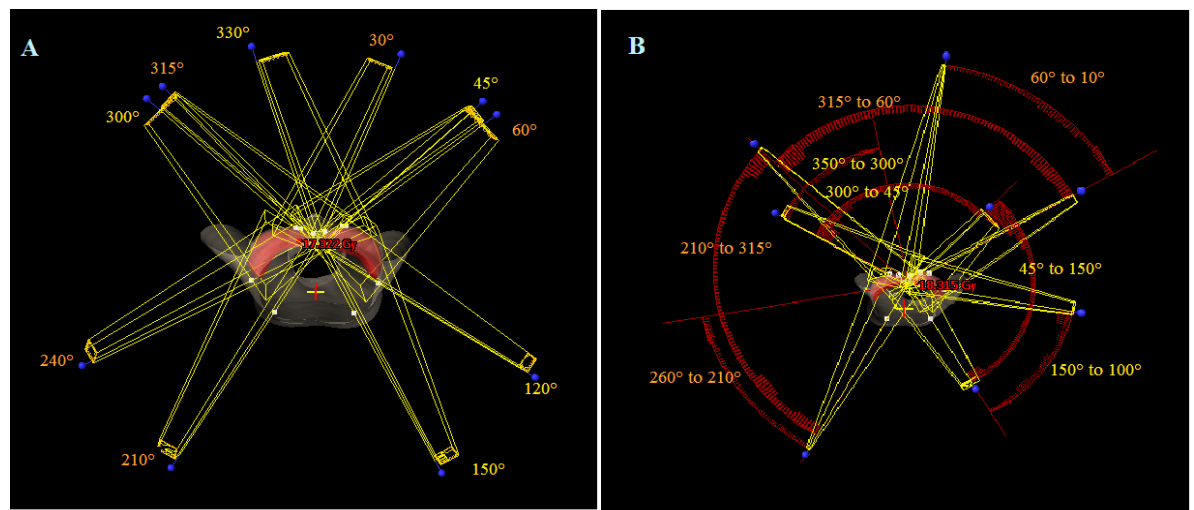

Figure 2. Field Arrangements in (a) IMRT Plan (5 fields for each breast), (b) VMAT Plan (2 partial and 2 tangential Arcs for Each Breast)

TT were significantly less in the 3DCRT+VMAT plan ( $\mathrm{p}$ $<0.00001)$. The plan quality score for the PTV has shown that the 3DCRT+VMAT and 3DCRT+IMRT plans were better than the IMRT+VMAT and VMAT+IMRT plans.

Table 3 summarizes the OARs dosimetric comparison results of all hybrid plans. On comparing doses to OARs, most of the dose parameters were showed statistically significant differences among the hybrid plans $(\mathrm{p}<0.05)$. The mean dose, $\mathrm{V}_{5 \mathrm{~Gy}}$, and $\mathrm{V}_{20 \mathrm{~Gy}}$ of both LL and RL were less in the 3DCRT \pm IMRT plan. Similarly, the heart mean dose, $\mathrm{V}_{5 \mathrm{~Gy}}$, and $\mathrm{V}_{25 \mathrm{~Gy}}$ were less in the 3DCRT+IMRT plan. The mean doses to LAD, RCA, esophagus, trachea, NT were significantly less in the 3DCRT+IMRT plan compared to other hybrid plans. The low dose levels $\left(\mathrm{V}_{5 \mathrm{~Gy}}\right)$ to lungs and heart were higher with VMAT+IMRT and IMRT+VMAT plans, whereas $\mathrm{V}_{35 \mathrm{~Gy}}$ of lungs and heart and $\mathrm{V}_{30 \mathrm{~Gy}}$ of LAD were less in the VMAT+IMRT plan. The overall plan quality scores that incorporate all dosimetric parameters (PTV and OARs) were 1.035, 0.912, 1.102, and 1.381 for 3DCRT+IMRT, 3DCRT+VMAT, IMRTVVMAT, and VMAT+IMRT plans respectively.

\section{Discussion}

RT of SBBC is more difficult than unilateral breast due to larger target volume and involvement of both lungs. The goal of this dosimetric comparison study was to evaluate the achievability of 4 different hybrid technique combinations, including 3DCRT+IMRT, 3DCRT+VMAT, IMRT+VMAT, and VMAT+IMRT for SBBC using a hypofractionated dose regimen. Clinical trials on the accelerated hypofractionated RT for breast cancer have been shown promising results. The UK Standardization of Breast Radiotherapy trial-A (START A) was explored 39 Gy and 41.6 Gy in 13 fractions over 5 -week dose regimens (Bentzen et al., 2008). While START B trial proposed a dose prescription of $40 \mathrm{~Gy}$ in 15 daily fractions over 3 weeks (Bentzen et al., 2008). These accelerated hypofractionated RT reduces the overall treatment course time and cost to the patients and reduces resource consumption.

3DCRT is the standard technique for breast cancer in most institutions worldwide. Nevertheless, the 3DCRT technique failed to provide better results for patients with SBBC. Precisely in the inter breast region, reducing the hotspot due to overlapping fields is a difficult task with the 3DCRT plan alone. However, the present hybrid planning study showed that the 3DCRT plan is optimal as a base-dose plan. The 3DCRT plan utilized lesser planning time, demanding fewer MUs compared to the IMRT and VMAT base-dose plans. In addition, the impact of setup and breathing movements are more in the IMRT/VMAT compared to the 3DCRT (Zhou et al., 2016). The 3DCRT beams are capable to accommodate the setup and shape changes of breasts with extended fields, along the skin surface region.

As part of the hybrid component, the IMRT and VMAT techniques are used to improve the discrepancies of the base-dose plans. Therefore the beam arrangement strategy of the IMRT and VMAT fields is more important in the hybrid setting. This study utilized 5 IMRT fields for each breast. Increasing the number of IMRT fields provides better results at the cost of increased MUs and treatment time. Consequently, patients with massive breast

Table 2. Dosimetric Parameters Results for Planning Target Volume and Delivery Efficiency

\begin{tabular}{lccccc}
\hline Parameter & 3DCRT+IMRT & 3DCRT+VMAT & IMRT+VMAT & VMAT+IMRT & p value* \\
\hline COI & $\mathbf{1 . 0 3 7} \pm \mathbf{0 . 0 1 0}$ & $1.042 \pm 0.009$ & $1.047 \pm 0.012$ & $1.045 \pm 0.007$ & $0.026 \#$ \\
CI & $1.179 \pm 0.039$ & $1.165 \pm 0.030$ & $1.180 \pm 0.038$ & $\mathbf{1 . 1 4 9} \pm \mathbf{0 . 0 3 5}$ & 0.068 \\
UI & $\mathbf{1 . 0 8 8} \pm \mathbf{0 . 0 2 2}$ & $1.093 \pm 0.018$ & $1.103 \pm 0.025$ & $1.110 \pm 0.019$ & $0.012 \#$ \\
GI & $\mathbf{2 . 0 5 3} \pm \mathbf{0 . 2 0 2}$ & $2.059 \pm 0.202$ & $\mathbf{2 . 0 5 3} \pm \mathbf{0 . 2 3 1}$ & $2.059 \pm 0.256$ & 0.991 \\
MU & $1264.9 \pm 124.7$ & $\mathbf{7 0 4 . 1} \pm \mathbf{2 3 . 9}$ & $950.0 \pm 124.9$ & $1491.9 \pm 203.7$ & $<0.001 \#$ \\
TT $(\min )$ & $9.12 \pm 0.41$ & $\mathbf{6 . 5 9} \pm \mathbf{0 . 0 4}$ & $7.49 \pm 0.24$ & $8.84 \pm 0.60$ & $<0.001 \#$ \\
\hline
\end{tabular}

COI, Coverage index; CI, Conformity index; UI, Uniformity index; GI, Gradient index; MU, Monitor unit; TT, Treatment time; Bold, Better result;

*, Kruskal-Wallis test; \#, statistically significant result 
Table 3. Comparison Results of Organs at Risk Dosimetric Parameters

\begin{tabular}{|c|c|c|c|c|c|}
\hline Parameter & 3DCRT+IMRT & 3DCRT+VMAT & IMRT+VMAT & VMAT+IMRT & p value* \\
\hline \multicolumn{6}{|l|}{ LL } \\
\hline $\mathrm{D}_{\text {Mean }}(\mathrm{Gy})$ & $6.39 \pm 0.41$ & $6.83 \pm 0.34$ & $6.72 \pm 0.54$ & $7.94 \pm 0.60$ & $<0.001^{\#}$ \\
\hline $\mathrm{V}_{5 \mathrm{~Gy}}(\%)$ & $34.14 \pm 4.35$ & $37.59 \pm 4.46$ & $36.40 \pm 3.73$ & $47.59 \pm 4.59$ & $<0.001^{\#}$ \\
\hline $\mathrm{V}_{20 \mathrm{~Gy}}(\%)$ & $8.18 \pm 1.75$ & $8.99 \pm 1.60$ & $8.77 \pm 2.25$ & $9.89 \pm 1.92$ & $0.046^{\#}$ \\
\hline $\mathrm{V}_{35 \mathrm{~Gy}}(\%)$ & $1.17 \pm 0.53$ & $2.01 \pm 0.62$ & $1.71 \pm 0.68$ & $0.85 \pm 0.58$ & $<0.001^{\#}$ \\
\hline \multicolumn{6}{|l|}{ RL } \\
\hline $\mathrm{D}_{\text {Mean }}(\mathrm{Gy})$ & $6.97 \pm 0.58$ & $7.91 \pm 0.70$ & $8.12 \pm 0.76$ & $8.70 \pm 0.99$ & $<0.001^{\#}$ \\
\hline $\mathrm{V}_{5 \mathrm{~Gy}}(\%)$ & $36.95 \pm 5.69$ & $45.10 \pm 6.50$ & $46.11 \pm 6.87$ & $52.57 \pm 5.41$ & $<0.001^{\#}$ \\
\hline $\mathrm{V}_{20 \mathrm{~Gy}}(\%)$ & $9.13 \pm 3.28$ & $10.20 \pm 3.10$ & $10.79 \pm 2.95$ & $10.91 \pm 3.24$ & 0.149 \\
\hline $\mathrm{V}_{35 \mathrm{~Gy}}(\%)$ & $2.18 \pm 1.34$ & $3.46 \pm 1.50$ & $2.89 \pm 1.69$ & $1.96 \pm 1.20$ & $0.032^{\#}$ \\
\hline \multicolumn{6}{|l|}{ Heart } \\
\hline $\mathrm{D}_{\text {Mean }}(\mathrm{Gy})$ & $3.62 \pm 0.45$ & $4.86 \pm 0.97$ & $5.08 \pm 1.22$ & $5.55 \pm 0.33$ & $<0.001^{\#}$ \\
\hline $\mathrm{V}_{5 \mathrm{~Gy}}(\%)$ & $12.10 \pm 3.59$ & $27.73 \pm 12.39$ & $30.65 \pm 16.52$ & $36.91 \pm 5.24$ & $<0.001^{\#}$ \\
\hline $\mathrm{V}_{25 \mathrm{~Gy}}(\%)$ & $2.18 \pm 0.57$ & $2.65 \pm 0.55$ & $2.22 \pm 0.44$ & $1.37 \pm 0.75$ & $<0.001^{\#}$ \\
\hline $\mathrm{V}_{35 \mathrm{~Gy}}(\%)$ & $0.29 \pm 0.28$ & $0.50 \pm 0.29$ & $0.47 \pm 0.23$ & $0.22 \pm 0.28$ & $0.002^{\#}$ \\
\hline \multicolumn{6}{|l|}{$\mathrm{LAD}$} \\
\hline $\mathrm{D}_{\text {Mean }}(\mathrm{Gy})$ & $13.56 \pm 2.60$ & $16.04 \pm 3.08$ & $15.90 \pm 2.62$ & $15.25 \pm 2.45$ & 0.069 \\
\hline $\mathrm{V}_{30 \mathrm{~Gy}}(\%)$ & $11.61 \pm 6.97$ & $19.45 \pm 7.50$ & $14.32 \pm 6.04$ & $6.88 \pm 7.04$ & $<0.001^{\#}$ \\
\hline \multicolumn{6}{|l|}{$\mathrm{RCA}$} \\
\hline $\mathrm{D}_{\text {Mean }}(\mathrm{Gy})$ & $3.35 \pm 0.55$ & $4.92 \pm 1.26$ & $5.75 \pm 2.07$ & $5.15 \pm 0.99$ & $0.001^{\#}$ \\
\hline \multicolumn{6}{|l|}{ Esophagus } \\
\hline $\mathrm{D}_{\text {Mean }}(\mathrm{Gy})$ & $2.32 \pm 1.54$ & $4.03 \pm 0.88$ & $4.12 \pm 1.29$ & $3.49 \pm 1.37$ & $0.002^{\#}$ \\
\hline \multicolumn{6}{|l|}{ Trachea } \\
\hline $\mathrm{D}_{\text {Mean }}(\mathrm{Gy})$ & $2.54 \pm 1.50$ & $4.62 \pm 1.04$ & $4.77 \pm 1.63$ & $3.90 \pm 1.63$ & $0.001^{\#}$ \\
\hline \multicolumn{6}{|l|}{ GEJCP } \\
\hline $\mathrm{D}_{\text {Mean }}(\mathrm{Gy})$ & $1.00 \pm 0.40$ & $1.23 \pm 0.47$ & $1.18 \pm 0.38$ & $1.19 \pm 0.49$ & 0.482 \\
\hline \multicolumn{6}{|l|}{$\mathrm{SC}$} \\
\hline $\mathrm{D}_{\mathrm{Max}}(\mathrm{Gy})$ & $2.73 \pm 1.85$ & $4.58 \pm 1.13$ & $4.31 \pm 1.09$ & $4.64 \pm 2.32$ & $0.016^{\#}$ \\
\hline \multicolumn{6}{|l|}{ NT } \\
\hline $\mathrm{D}_{\text {Mean }}(\mathrm{Gy})$ & $4.22 \pm 0.51$ & $4.28 \pm 0.45$ & $4.22 \pm 0.43$ & $4.74 \pm 0.66$ & 0.086 \\
\hline $\mathrm{V}_{5 \mathrm{GV}}(\%)$ & $17.74 \pm 3.03$ & $18.13 \pm 2.39$ & $18.30 \pm 2.61$ & $23.04 \pm 3.39$ & $0.001^{\#}$ \\
\hline
\end{tabular}

LL, Left lung; RL, Right lung; LAD, Left anterior descending coronary artery; RCA, Right coronary artery; GEJCP, Coeliac plexus and gastroesophageal junction; SC, Spinal cord; NT, Normal tissue; Gy, Gray; $\mathrm{D}_{\text {Mean }}$, Mean dose; $\mathrm{D}_{\text {Max }}$, Maximum dose; $\mathrm{V}_{\mathrm{XGy}}$, Volume receiving X dose; Bold, Better result; *, Kruskal-Wallis test; \#, statistically significant result

volume might require 7 fields of IMRT to get better beam modulation. For the VMAT plan, different arc designs like split arcs (Boman et al., 2016), multiple partial arcs (Tsai et al., 2012), modified VMAT (Lai et al., 2016), tangential VMAT (Viren et al., 2015) have been explored in the literature. The purpose of the split arcs or multiple partial arcs is to reduce the size of the arc field so that it will reduce the modulation complexity by reducing the MLC travel range. The use of the tangential arc is to reduce the dose to contralateral OARs and to reduce the low dose levels to NT. The present study employed two split arcs and two tangential arcs for each breast and the field size was limited to $15 \mathrm{~cm}$ to increase the smooth delivery of MLC.

Previous studies reported the dosimetric comparison of hybrid techniques and individual IMRT, VMAT techniques in SBBC RT (Gadea et al., 2021; Huang et al., 2019; Kim et al., 2018; Subramanian et al., 2016). In a dosimetric comparison study, Huang et al., (2019) evaluated fixedjaw IMRT and tangential partial VMAT in SBBC using a hypofractionated dose of 42.56 Gy in 16 fractions. The results showed that the IMRT was better in terms of target coverage and OAR sparing at low dose levels, whereas the VMAT was superior at the high dose levels. Similarly, Kim et al., (2018) compared IMRT, VMAT, and 3DCRT on SBBC patients. The dose prescription was 50 Gy in 25 fractions. The IMRT plan showed superior results in most of the dosimetric parameters, while VMAT presented better treatment efficiency with less MU and TT. The present study also showed a similar trend with 3DCRT+IMRT and 3DCRT+VMAT hybrid plans.

Subramanian et al., (2016) evaluated VMAT and hybrid VMAT in SBBC using a conventional dose schedule. It was reported that the hybrid VMAT was superior compared to VMAT. The present study was intended to compare different hybrid techniques for 
further improvement in SBBC RT planning using a hypofractionated dose regimen. Gadea et al., (2021) compared the feasibility of different dose regimens for SBBC using the VMAT technique. For the same dose schedule, the mean dose to heart achieved in the Gadea et al. study (VMAT) and the present study (3DCRT+VMAT) were 4.8 Versus 4.86 Gy respectively. Similarly, the mean lungs doses were 9.8 versus $7.3 \mathrm{~Gy}$ and $\mathrm{V}_{20 \mathrm{~Gy}}$ of lungs were 12.1 versus $9.6 \%$ in Gadea et al study and the present study respectively.

Gaudino et al., (2018) compared VMAT to SBBC RT in deep inspiration breath-hold (DIBH) versus free-breathing set up. While it was reported that the DIBH method can able to reduce the dose to the heart, LAD, and lungs, this practice might not be achievable for all patients. The TT in SBBC irradiation was twice as compared to unilateral breast RT and show concerns in the breath-hold constancy, reduction of patient movement during treatment, and patient's comfort. Therefore, the necessity of DIBH should be evaluated in a clinical setting for each patient. The exclusion of nodal stations was the limitation of this study. However, further investigation including nodal stations is required, which could be anticipated in some clinical settings.

The present dosimetric study revealed that all hybrid plans provided quality results for SBBC irradiation. The 3DCRT+IMRT technique provided superior results for most of the dosimetric parameters. The 3DCRT+VMAT technique showed superior results in terms of fewer MU and delivery time and the integrated plan quality matrix favored the same. Further, bearing in mind the uniqueness of each patient, the trade-off between 3DCRT+VMAT and 3DCRT+IMRT needs to be evaluated with regards to plan complexity, OAR sparing and planning time in a clinical setting. Further research involving different beam arrangements for IMRT and VMAT is warranted for improved outcomes.

\section{Author Contribution Statement}

The contributions of all authors must be described in the following manner: The authors confirm contribution to the paper as follows: study conception and design: $\mathrm{K}$. Balaji, V. Ramasubramanian; data collection: K. Balaji; analysis and interpretation of results: K. Balaji; draft manuscript preparation: K. Balaji, V. Ramasubramanian. All authors reviewed the results and approved the final version of the manuscript.

\section{Acknowledgments}

The authors would like to thank Dr. Balajisubramanian S, Dr. Balasundaram V and Dr. Sathiya K, Radiation Oncologists and Mr. Moorthi T, Medical Physicist of Gleneagles Global Hospitals, Chennai, for their helpful discussion and advice.

\section{References}

Badiyan SN, Shah C, Arthur D, et al (2014). Hypofractionated regional nodal irradiation for breast cancer: examining the data and potential for future studies. Radiother Oncol, 110, 39-44.

Balaji K, Subramanian B, Yadav P, Anu Radha C, Ramasubramanian V (2016). Radiation therapy for breast cancer: Literature review. Med Dosim, 41, 253-7.

Balaji K, Yadav P, BalajiSubramanian S, Anu Radha C, Ramasubramanian V (2018). Hybrid volumetric modulated arc therapy for chest wall irradiation: For a good plan, get the right mixture. Phys Med, 52, 86-92.

Bahrainy M, Kretschmer M, Jost V, et al (2016). Treatment of breast cancer with simultaneous integrated boost in hybrid plan technique. Strahlenther Onkol, 192, 333-41.

Bentzen SM, Agrawal RK, Aird EG, et al (2008). The UK standardisation of breast radiotherapy (START) Trial A of radiotherapy hypofractionation for treatment of early breast cancer: a randomised trial. Lancet, 9, 331-41.

Bentzen SM, Agrawal RK, Aird EG, et al (2008). The UK standardisation of breast radiotherapy (START) Trial B of radiotherapy hypofractionation for treatment of early breast cancer: a randomised trial. Lancet, 371, 1098-107.

Boman E, Rossia M, Haltamo M, Skyttä T, Kapanen M (2016). A new split arc VMAT technique for lymph node positive breast cancer. Phys Med, 32, 1428-36.

Chen YG, Li AC, Li WY, et al (2017). The feasibility study of a hybrid coplanar arc technique versus hybrid intensity-modulated radiotherapy in treatment of early-stage left-sided breast cancer with simultaneous-integrated boost. J Med Phys, 42, 1-8.

Gadea J, Ortiz I, Roncero R, et al (2021). Synchronous bilateral breast cancer treated with a 3-week hypofractionated radiotherapy schedule: clinical and dosimetric outcomes. Clin Transl Oncol, 23, 1915-22.

Gaudino D, Cima S, Frapolli M, et al (2018). Volumetric modulated arc therapy applied to synchronous bilateral breast cancer radiotherapy: dosimetric study on deep inspiration breath hold versus free breathing set up. Biomed Phys Eng Express, 4, 045007.

Haviland JS, Owen RJ, Dewar JA, et al (2013). The UK Standardisation of Breast Radiotherapy (START) trials of radiotherapy hypofractionation for treatment of early breast cancer: 10-year follow-up results of two randomised controlled trials. Lancet Oncol, 4, 1086-94.

Huang JH, Wu XX, Lin X, et al (2019). Evaluation of fixed-jaw IMRT and tangential partial-VMAT radiotherapy plans for synchronous bilateral breast cancer irradiation based on a dosimetric study. J Appl Clin Med Phys, 20, 31-41.

Jost V, Kretschmer M, Sabatino M, et al (2015). Heart dose reduction in breast cancer treatment with simultaneous integrated boost: comparison of treatment planning and dosimetry for a novel hybrid technique and 3D-CRT. Strahlenther Onkol, 191, 734-41.

Khan AJ, Poppe MM, Goyal S, et al (2017). Hypofractionated postmastectomy radiation therapy is safe and effective: first results from a prospective phase II trial. J Clin Oncol, 35, 2037-43.

Kheirelseid EA, Jumustafa H, Miller N, et al (2011). Bilateral breast cancer: analysis of incidence, outcome, survival and disease characteristics. Breast Cancer Res Treat, 126, 131-40.

Kim SJ, Lee MJ, Youn SM (2018). Radiation therapy of synchronous bilateral breast carcinoma (SBBC) using multiple techniques. Med Dosim, 43, 55-68.

Lai Y, Chen Y, Wu S, et al (2016). Modified volumetric modulated arc therapy in left sided breast cancer after radical mastectomy with flattening filter free versus flattened beams. Medicine, 95, e3295.

Lin J, Yeh D, Yeh H, Chang CF, Lin JC (2015). Dosimetric 
comparison of hybrid volumetric-modulated arc therapy, volumetric-modulated arc therapy and intensity-modulated radiation therapy for left-sided early breast cancer. Med Dosim, 40, 262-7.

Narasimhulu BC, Valiyaveettil D, Joseph D, et al (2020). Synchronous bilateral breast cancer patients treated with hypofractionated bilateral breast irradiation: A dosimetric and clinical study. $J$ Cancer Res Ther, 16, 1309-13.

Scorsetti M, Alongi F, Fogliata A, et al (2012). Phase I-II study of hypofractionated simultaneous integrated boost using volumetric modulated arc therapy for adjuvant radiation therapy in breast cancer patients: a report of feasibility and early toxicity results in the first 50 treatments. Radiat Oncol, 7, 145.

Subramanian SB, Balaji K, Thirunavukarasu M, Premkumar S (2016). Bilateral breast irradiation using hybrid volumetric modulated arc therapy (h-VMAT) technique: A Planning Case Report. Cureus, 8, e914.

Tsai PF, Lin SM, Lee SH, et al (2012). The feasibility study of using multiple partial volumetric-modulated arcs therapy in early stage left-sided breast cancer patients. $J$ Appl Clin Med Phys, 13, 62-73.

Viren T, Heikkilä J, Myllyoja K, et al (2015). Tangential volumetric modulated arc therapy technique for left-sided breast cancer radiotherapy. Radiat Oncol, 10, 79.

Zhou S, Zhu X, Zhang M, et al (2016). Estimation of internal organ motion-induced variance in radiation dose in non-gated radiotherapy. Phys Med Biol, 61, 8157-79.

\section{cc) (i) (8)}

This work is licensed under a Creative Commons AttributionNon Commercial 4.0 International License. 